\title{
Challenges of monitoring use of secondary care at local level: a study based in London, UK
}

\author{
Laurent Chenet, Martin McKee
}

\begin{abstract}
Study objective - To provide those working at district level with practical guidance on using hospital data linked to small geographic areas to explore patterns of care. Design - Examination of the association between age standardised hospital episode rates for the commonest diagnostic categories and deprivation levels (Carstairs index) of the electoral ward of residence. Setting - An inner London district, Kensington, Chelsea and Westminster, with a population of approximately 325000 .

Population - All finished consultant episodes recorded in NHS hospitals for the district population in the year to April 1994.

Main results - Many, but not all, disease categories were associated strongly with deprivation, with high episode rates in the most deprived electoral wards. This is partly due to more of those in deprived areas being admitted to hospital and to them being admitted more often.

Conclusions - A wide range of factors needs to be taken into account in interpreting these data. They include the contribution of the private sector and artifacts of both the numerator and denominator. This paper provides a framework for those working at district level to begin to analyse the association between hospitalisation and deprivation locally. It also identifies some of the issues that must be taken into account when seeking to interpret these data.
\end{abstract}

(f Epidemiol Community Health 1996;50:359-365)

Health authorities in the reformed British NHS are responsible for assessing the health needs of their population and ensuring that a range of appropriate and effective services is in place to meet these. ${ }^{1}$ The recent work designed to produce a new health care resource allocation formula ${ }^{2}$ has shown that uptake of secondary care at a population level in the United Kingdom varies and is associated with differences in socioeconomic variables. Routine hospital data linked by postcode to small geographical areas and thus to measures of deprivation is one way in which the outcome of this process may be monitored in respect of secondary inpatient care.

This paper uses a case study in one district to examine some of the methodological challenges involved. We describe an examination of admission rates for the commonest specific diag- noses in an inner London health district Kensington, Chelsea and Westminster. The district has a population of approximately 325000 . It is extremely heterogeneous; some parts are very poor while others, such as Knightsbridge, are home to some of the wealthiest individuals in the country. In addition, a high proportion of the population is from minority ethnic groups. The district is, however, well served by hospitals, with three major teaching hospitals and several special health authorities either within its boundaries or nearby.

\section{Methods}

The study examined hospital episode data on admissions to all NHS hospitals anywhere in England for residents of Kensington, Chelsea, Westminster Commissioning Agency for the year to April 1994. To overcome the problems of analysis arising from the very many combinations of codes in the International Classification of Disease (ICD), diagnostic data were condensed to generate one of 400 disease categories, using the American Disease Staging system. ${ }^{3}$ An evaluation of disease staging ${ }^{3}$ using British data has been described elsewhere. ${ }^{4}$

The number of episodes in each disease category was ranked. Those containing more than 400 episodes, those with specific labels (that is, not, for example, "other urinary tract disorders"), and those not related to obstetrics were selected for further study (table 1). This accounted for $24 \%$ of all episodes. By mapping on the basis of postcodes, episodes were assigned to an electoral ward of residence. A composite measure of deprivation, the Carstairs index, ${ }^{5}$ was obtained from census data for each ward. This summates measures of unemployment, overcrowding, no car ownership and social class.

The number of episodes in each of the disease categories being studied that arose from each ward was identified and applied to the resident population of the ward as measured in the 1991 census. An age standardised episode ratio (SER) for each ward was calculated by applying the overall age specific (in five year age bands) rates for the district to the age distribution in each ward to yield an expected number of episodes from each ward. The standardised ratio was then calculated by dividing the observed by the expected rates and multiplying by 100 .

The association at ward level between the episode ratio and the deprivation score was calculated by regression using Spearman's correlation method. To illustrate the size of any effect, for those disease categories for which 
Table 1 Commonest disease categories in hospital episode data for Kensington, Chelsea, Westminster residents: 1993-94

\begin{tabular}{llll}
\hline Disease category & Frequency & $\%$ of total & Cumulative \% \\
\hline Delivery, vaginal & 3058 & $4 \cdot 9$ & $4 \cdot 9$ \\
Other urinary tract disorders & 3000 & $4 \cdot 8$ & $9 \cdot 7$ \\
Other general conditions & 1816 & $2 \cdot 9$ & $12 \cdot 6$ \\
Abortion, therapeutic & 1793 & $2 \cdot 8$ & $15 \cdot 4$ \\
Ante- \& postpartum conditions & 1698 & $2 \cdot 7$ & $18 \cdot 1$ \\
Other respiratory conditions & 1608 & $2 \cdot 6$ & $20 \cdot 7$ \\
Other gastrointestinal conditions & 1575 & $2 \cdot 5$ & $23 \cdot 2$ \\
Other musculoskeletal conditions & 1474 & $2 \cdot 3$ & $25 \cdot 5$ \\
Coronary artery disease & 1434 & $2 \cdot 3$ & $27 \cdot 8$ \\
Other cardiovascular conditions & 1238 & $2 \cdot 0$ & $29 \cdot 8$ \\
Other eye, ENT conditions & 1204 & $1 \cdot 9$ & $31 \cdot 7$ \\
Spontaneous abortion & 1204 & $1 \cdot 9$ & $33 \cdot 6$ \\
Other skin conditions & 1166 & $1 \cdot 8$ & $35 \cdot 4$ \\
Birth trauma \& residual disorders & 948 & $1 \cdot 5$ & $36 \cdot 9$ \\
Other neoplasms & 948 & $1 \cdot 5$ & $38 \cdot 4$ \\
Abdominal pain & 851 & $1 \cdot 4$ & $39 \cdot 8$ \\
Cataract & 843 & $1 \cdot 3$ & $41 \cdot 1$ \\
Cerebrovascular disease & 786 & $1 \cdot 2$ & $42 \cdot 3$ \\
Bronchitis and COPD & 786 & $1 \cdot 2$ & $43 \cdot 5$ \\
Other nervous system disorders & 752 & $1 \cdot 2$ & $44 \cdot 7$ \\
Acute renal failure & 684 & $1 \cdot 1$ & $45 \cdot 8$ \\
Bursitis & 615 & $1 \cdot 0$ & $46 \cdot 8$ \\
Asthma & 609 & $1 \cdot 0$ & $47 \cdot 8$ \\
Other traumas & 604 & $1 \cdot 0$ & $48 \cdot 8$ \\
Other conditions of the female genital system & 598 & $0 \cdot 9$ & $49 \cdot 7$ \\
Hernia (external) & 573 & $0 \cdot 9$ & $50 \cdot 6$ \\
Functional digestive disorders & 558 & $0 \cdot 9$ & $51 \cdot 5$ \\
Cancer of the colon & 537 & $0 \cdot 9$ & $52 \cdot 4$ \\
Head injury & 535 & $0 \cdot 8$ & $53 \cdot 2$ \\
Bacterial pneumonia & 510 & $0 \cdot 8$ & 54 \\
Cancer of the breast & 501 & $0 \cdot 8$ & $54 \cdot 8$ \\
Cancer at genitourinary sites - bladder & 482 & $0 \cdot 8$ & $55 \cdot 6$ \\
Gastritis & 447 & $0 \cdot 7$ & $56 \cdot 3$ \\
Dysfunctional uterine bleeding & 441 & $0 \cdot 7$ & 57 \\
UTI (other than tuberculosis) & 434 & $0 \cdot 7$ & $57 \cdot 7$ \\
Fracture of the hip & 434 & $0 \cdot 7$ & $58 \cdot 4$ \\
Peptic ulcer & 423 & $0 \cdot 7$ & $59 \cdot 1$ \\
Dental disease & 402 & $0 \cdot 6$ & $59 \cdot 7$ \\
Epilepsy/convulsion & 401 & $0 \cdot 6$ & $60 \cdot 3$ \\
\hline COPD chronicobstren & & \\
\end{tabular}

$\mathrm{COPD}=$ chronic obstructive pulmonary disease; $\mathrm{ENT}=$ ear, nose and throat; UTI = urinary trac infection.

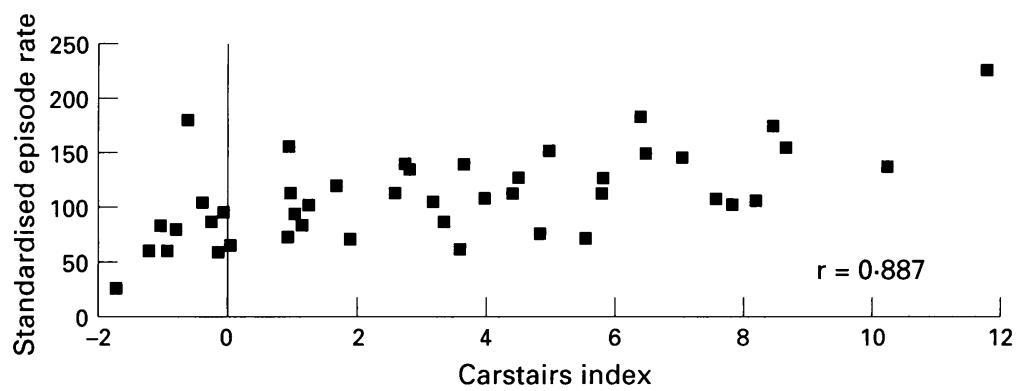

Figure 1 Relation between Carstairs index and age standardised episode rate.

Table 2 Correlation between Carstairs index and standardised episode ratio for commonest disease categories

\begin{tabular}{llc}
\hline Disease category & $\begin{array}{l}\text { Spearman's rank } \\
\text { correlation }\end{array}$ & $\begin{array}{c}\text { Significance } \\
\text { (two tailed) }\end{array}$ \\
\hline Abdominal pain & 0.6696 & $<0.0001$ \\
Acute renal failure & 0.3298 & 0.029 \\
Asthma & 0.5102 & $<0.0001$ \\
Bacterial pneumonia & $0 \cdot 2927$ & 0.054 \\
Bronchitis and COPD & $0 \cdot 7174$ & $<0.0001$ \\
Bursitis & $0 \cdot 7281$ & $<0.0001$ \\
Cancer at genitourinary sites - bladder & 0.5181 & $<0.0001$ \\
Cancer of the breast & 0.082 & 0.597 \\
Cancer of the colon & 0.1696 & 0.271 \\
Cataract & 0.3549 & 0.018 \\
Cerebrovascular disease & 0.5175 & $<0.0001$ \\
Coronary artery disease & 0.5548 & $<0.0001$ \\
Dental disease & 0.7333 & $<0.0001$ \\
Dysunctional uterine bleeding & 0.3955 & 0.008 \\
Epilepsy/convulsion & 0.516 & $<0.0001$ \\
Fracture of the hip & 0.3357 & 0.026 \\
Functional digestive disorders & 0.7752 & $<0.0001$ \\
Gastritis & 0.5369 & $<0.0001$ \\
Head injury & 0.1452 & 0.347 \\
Hernia (external) & 0.5157 & $<0.0001$ \\
Peptic ulcer & 0.5212 & $<0.0001$ \\
Spontaneous abortion & 0.7049 & $<0.0001$ \\
Therapeutic aborting & 0.7917 & $<0.0001$ \\
UTI (other than tuberculosis) & 0.5442 & $<0.0001$ \\
\hline
\end{tabular}

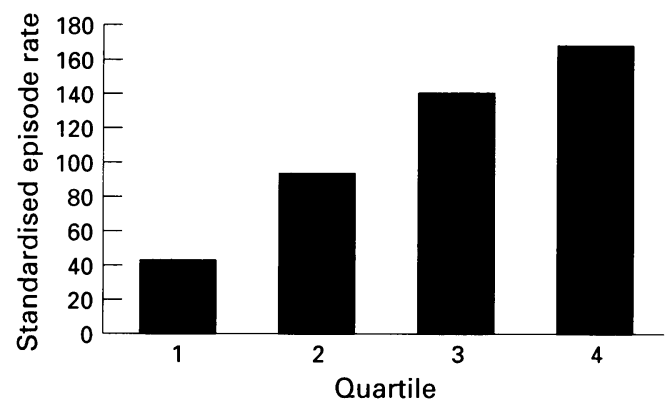

Figure 2 Steady rise: bronchitis and chronic obstructive pulmonary disease.

there was a statistically significant association with deprivation, the age standardised admission rates have been calculated for groups of wards divided into quartiles in terms of the Carstairs index.

An attempt was made to match episodes to people by aggregating records that had matching dates of birth, principal disease categories, sex and postcodes. This generated pseudopatients. These data were then used to assess the impact of differences in the ratio of episodes to people by calculating the person-based annual hospitalisation rate by electoral ward, calculating the ratio of episodes to pseudo-patients by deprivation quartiles and, for selected disease categories, regression the person-based annual hospitalisation rate against the Carstairs index.

All analyses were undertaken using either SPSS for Windows or Excel. ${ }^{7}$

\section{Results}

There was a strong correlation between deprivation and SER for all diagnoses together (fig 1) $(r=0.887, p<0.0001)$. The SER in the poorest quartile of wards was twice as high as that in the wealthiest one $(132.5$ and 66.5 respectively).

The correlations between SERs and Carstairs indices for each disease category are shown in table 2 . In nearly every case the association was statistically significant, although in all cases the strength of the association was less than that for all diagnoses together. The strongest associations were seen for therapeutic abortions and functional digestive disorders, although the association was even stronger when all disease categories other than those listed in table 2 were regressed against Carstairs indices $(r=$ $0 \cdot 8646, \mathrm{p}<0 \cdot 0001$ ).

The SERs in each deprivation quartile are shown in table 3. The ratio of the SER in the most and least deprived quartiles was typically between 1.5 and 3.5 but was almost 7 for functional digestive disorders. In this category, the commonest primary diagnoses were dyspepsia $(50 \%)$, constipation $(20 \%)$, and other functional disorders of the intestine $(22 \%)$. In most of the conditions for which the correlation is significant, there is, as expected, a progressive increase in SER in each deprivation quartile. The shape of the trend varies, however, and three examples of typical patterns - what we 
Table 3 Standardised episode rate (numbers of episodes in parentheses) in electoral wards aggregated by level of deprivation

\begin{tabular}{llrlrl}
\hline & \multicolumn{2}{l}{ Deprivation quartile* } & & \\
\cline { 2 - 5 } & 1 & 2 & 3 & 4 & Ratio Q4Q1 \\
\hline Abdominal pain & $59(104)$ & $97(192)$ & $118(222)$ & $130(324)$ & $2 \cdot 2$ \\
Acute renal failure & $51(74)$ & $128(186)$ & $72(102)$ & $163(321)$ & $3 \cdot 2$ \\
Asthma & $69(87)$ & $88(116)$ & $114(144)$ & $129(259)$ & $1 \cdot 9$ \\
Bacterial pneumonia & $60(63)$ & $119(101)$ & $151(121)$ & $140(176)$ & $2 \cdot 3$ \\
Bronchitis and COPD & $43(74)$ & $92(134)$ & $141(198)$ & $168(356)$ & $3 \cdot 9$ \\
Bursitis & $44(59)$ & $99(133)$ & $105(136)$ & $161(280)$ & $3 \cdot 7$ \\
Cancer at genito urinary sites - bladder & $54(59)$ & $154(137)$ & $108(92)$ & $149(181)$ & $2 \cdot 8$ \\
Cancer of breast & $79(89)$ & $119(148)$ & $87(101)$ & $114(155)$ & $1 \cdot 4$ \\
Cancer of colon & $76(93)$ & $91(100)$ & $113(120)$ & $151(216)$ & $2 \cdot 0$ \\
Cataract & $74(138)$ & $135(199)$ & $152(214)$ & $118(243)$ & $1 \cdot 6$ \\
Cerebrovascular disease & $71(121)$ & $122(170)$ & $135(180)$ & $135(259)$ & $1 \cdot 9$ \\
Coronary artery disease & $62(202)$ & $138(401)$ & $115(323)$ & $127(485)$ & $2 \cdot 0$ \\
Dental disease & $49(39)$ & $60(52)$ & $114(92)$ & $139(218)$ & $2 \cdot 8$ \\
Dysfunctional uterine bleeding & $79(80)$ & $84(94)$ & $106(107)$ & $128(158)$ & $1 \cdot 6$ \\
Epilepsy & $65(54)$ & $89(74)$ & $125(99)$ & $133(166)$ & $2 \cdot 0$ \\
Fracture of the hip & $96(88)$ & $147(90)$ & $184(106)$ & $120(113)$ & $1 \cdot 3$ \\
Functional digestive disorders & $28(34)$ & $87(101)$ & $98(108)$ & $192(298)$ & $6 \cdot 9$ \\
Gastritis & $81(79)$ & $73(67)$ & $122(108)$ & $149(182)$ & $1 \cdot 8$ \\
Head injury & $90(100)$ & $86(100)$ & $136(151)$ & $107(168)$ & $1 \cdot 2$ \\
Hernia (external) & $62(77)$ & $107(128)$ & $134(154)$ & $124(202)$ & $2 \cdot 0$ \\
Peptic ulcer & $83(78)$ & $86(76)$ & $123(104)$ & $139(161)$ & $1 \cdot 7$ \\
Spontaneous abortion & $52(126)$ & $67(207)$ & $94(269)$ & $165(600)$ & $3 \cdot 2$ \\
Therapeutic abortion & $48(168)$ & $79(367)$ & $97(424)$ & $154(833)$ & $3 \cdot 2$ \\
UTI & $69(61)$ & $103(82)$ & $153(116)$ & $134(155)$ & $1 \cdot 9$ \\
\hline
\end{tabular}

Quartile 1 - least deprived, quartile 4 - most deprived.

have termed steady rise, rise and fall, and plateau - are shown in figures 2-4. It is likely, on the basis of the observed distributions, that they could be more appropriately described by a curvilinear relationship. This exercise would be helpful in generating hypotheses for the observed associations. For example, the decreased SER for cataract in the most deprived quartile could indicate a failure to recognise impaired vision in the primary care setting.

The median number of episodes per pseudopatient was one, except for cancer at genitourinary sites - bladder, where it was two. In all cases the maximum number of episodes per pseudo-patient was greater than one, typically between three and 10, but as high as 31 for cancer of the colon and 192 for acute renal

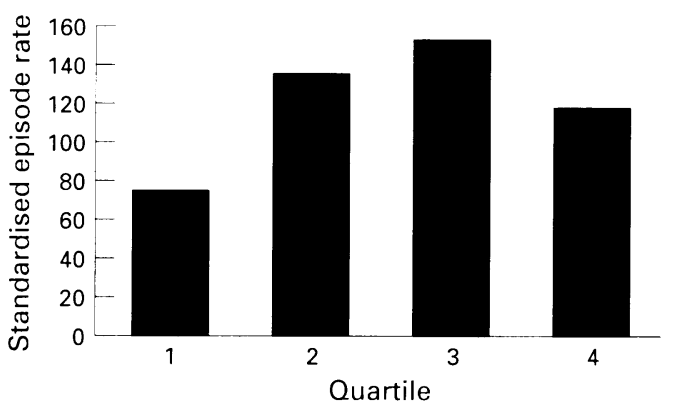

Figure 3 Rise and fall: cataract.

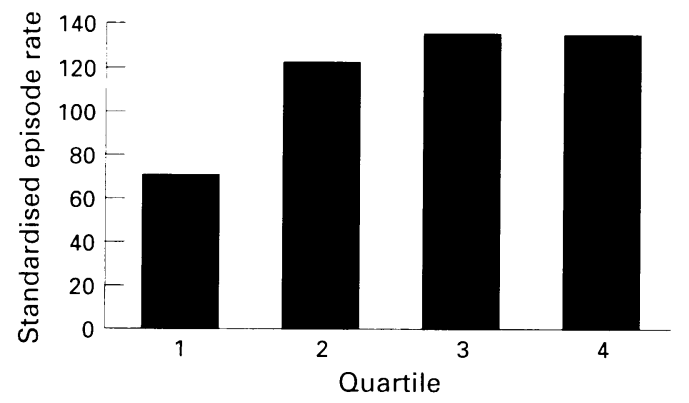

Figure 4 Plateau: cerebrovascular disease. failure. The ratio of episodes per pseudopatient in the wealthiest and poorest quartiles of wards is shown in table 4. For most disease categories the ratio was higher in poorer areas than in wealthier ones, suggesting that not only are more of the poor admitted but also that they are admitted more often. The exceptions included categories where there is a large discretionary element such as dental disease and gastritis. Again, this analysis raises questions where the answer is not readily apparent that could be explored in more detail, such as the greater numbers of episodes per pseudo-patient for some disease categories such as spontaneous abortion.

As expected, given the effect of deprivation level on the ratio of episodes to pseudo-patients, in those cases where further regression was undertaken the association was less strong than with episode based data. For example, for acute renal failure the correlation coefficient fell from 0.3298 to 0.06 , which, when taken with the figures for episode per pseudo-patient, suggest that the observed effect is largely due to those in more deprived wards being admitted more often. For asthma, there is still a decline, from 0.5102 to 0.369 but the correlation remains significant $(\mathrm{p}=001)$ suggesting that both factors are at work.

\section{Discussion}

This case study is consistent with the existing body of evidence that hospitalisation rates are associated with socioeconomic factors. It differs from much previous work in that it examines a range of specific diagnoses. The most striking features are, on one hand, the wide range of conditions that are associated with deprivation and, on the other hand, that this is not a general phenomenon and that some are not. These observations raise a range of questions for those involved in monitoring uptake of services. The remainder of this paper examines some of the possible explanations. These include the effect of the private sector, concerns 
Table 4 Ratio of episodes to pseudo-patients in least (Q1) and most (Q4) deprived quartiles of wards

\begin{tabular}{|c|c|c|}
\hline Disease category & $\begin{array}{l}\text { Episodes/ } \\
\text { pseudo-patient } \\
\text { Q1 }\end{array}$ & $\begin{array}{l}\text { Episodes/ } \\
\text { pseudo-patient } \\
\text { Q4 }\end{array}$ \\
\hline Abdominal pain & 1.09 & 1.09 \\
\hline Acute renal failure & $3 \cdot 0$ & $5 \cdot 1$ \\
\hline Asthma & $0 \cdot 24$ & $1 \cdot 34$ \\
\hline Bacterial pneumonia & $1 \cdot 14$ & $1 \cdot 16$ \\
\hline Bronchitis and COPD & 1.53 & 1.63 \\
\hline Bursitis & 1.04 & $1 \cdot 32$ \\
\hline Cancer at genitourinary sites - bladder & $2 \cdot 03$ & $2 \cdot 25$ \\
\hline Cancer of the breast & $2 \cdot 23$ & $2 \cdot 77$ \\
\hline Cancer of the colon & $3 \cdot 16$ & $3 \cdot 41$ \\
\hline Cataract & 1.09 & 1.09 \\
\hline Cerebrovascular disease & $1 \cdot 29$ & $1 \cdot 27$ \\
\hline Coronary artery disease & $1 \cdot 31$ & 1.46 \\
\hline Dental disease & $1 \cdot 11$ & 1.02 \\
\hline Dysfunctional uterine bleeding & 1.08 & $1 \cdot 07$ \\
\hline Epilepsy/convulsion & $1 \cdot 2$ & $1 \cdot 31$ \\
\hline Fracture of the hip & $1 \cdot 1$ & $1 \cdot 14$ \\
\hline Functional digestive disorders & 1.03 & 1.08 \\
\hline Gastritis & $1 \cdot 22$ & 1.06 \\
\hline Head injury & 1.04 & $1 \cdot 08$ \\
\hline Hernia (external) & 1.05 & $1 \cdot 07$ \\
\hline Peptic ulcer & $1 \cdot 22$ & $1 \cdot 2$ \\
\hline Spontaneous abortion & $1 \cdot 16$ & $1 \cdot 32$ \\
\hline Therapeutic abortion & 1.08 & $1 \cdot 1$ \\
\hline UTI (other than tuberculosis) & 0.09 & $1 \cdot 24$ \\
\hline
\end{tabular}

about the accuracy of the denominator (census under-enumeration) and the numerator (episode inflation), possible confounding by distance and social variation in diagnosis, as well as a true effect which could be due to differences in prevalence of disease, in the extent of timely treatment that could avoid admission, and in the effect of social factors on the need for admission. It concludes with some caveats concerning interpretation of this type of analysis and suggestions for further research.

\section{POTENTIAL ARTIFACTS}

The observation that hospitalisation rates are lower in wealthier areas could be due to diversion of admissions to the private sector, which is thought to account for about $20 \%$ of admissions in London. ${ }^{8}$ This could explain some of the associations noted, such as those for cataract and dental diseases. For example, if $20 \%$ of all cataract operations on this population were done in the private sector and these were exclusively among those living in the wealthiest quartile of wards, the SER for this section of the population would exceed that in the poorest quartile. Given the emphasis on elective surgery in private medicine, this is less likely to be the case for many of the other conditions.

The high rates in deprived areas might arise from under enumeration of the population denominator. In the most deprived wards in the district, up to $8 \%$ of those in certain age groups did not complete a form in the 1991 census. Modelling of the effect of this suggests that it is unlikely, on its own, to account for the large variations seen.

There is some evidence that episode inflation ${ }^{10}$ has selectively increased the numerator in some wards. As noted above, the episode rate combines the number of people admitted and the frequency with which they are admitted. In this case study the analysis has not been pursued in great detail because of the potential problems that cannot be easily re- solved using the methods of analysis available at district level and with the available data. The method of linking to create pseudo-patients appears reasonably robust and less than $0 \cdot 1 \%$ of postcode, date of birth and sex variables were missing. It is, however, possible (or even probable) that some are incorrect. There is also the problem of mobility in inner city districts where typically up to $20 \%$ of people change their address each year, not to mention the many homeless. As the number of events in many wards is already quite low, serious biases may arise from uncritical use of this approach.

If episode rates are used, some other issues must be taken into account. For example, there may be differences in the extent to which hospitals serving different parts of the district inflate episodes. Among the hospitals in the data set the ratio of episodes to pseudo-patients was typically 1.05 to 1.1 but it was 1.68 in one hospital, although it treats only a few patients from the district, most of whom are tertiary referrals. There are various reasons why these ratios are greater than one. One example is using a series of admissions over a short period instead of keeping a patient in hospital for an entire course of treatment. A second, seen with acute renal failure, is misclassification of some regular day attenders as inpatients, such as the patient with 192 recorded episodes in the year. In passing it should be noted that the health authority was paying for each attendance at the average specialty cost for a nephrology episode.

In this analysis the linkage included disease category. A more complicated problem arises when a patient has a series of episodes in a single admission but these are allocated different principal diagnoses and thus disease categories. These may or may not be related, such as where the first episode is labelled "chest pain" and subsequent ones are "ischaemic heart disease". Other situations may be less clear cut. There are several possible approaches to this problem but none is perfect. One is to include only the first recorded episodes in a hospital spell but, as in the case of "chest pain", this may produce a large number of non-specific diagnoses for those patients who are admitted briefly under the admitting general medical team and then transferred to a specialist unit. This is especially likely in cardiology or geriatric medicine. The final spell in a hospital episode might also be used but this would require a complex algorithm to identify these. Neither approach would capture the complexity of multiple admissions. There is no real alternative other than to examine the data in detail in each case, enabling situations such as that seen in the data with acute renal failure to be identified.

The findings could also be affected by bias arising from differential access if the poorer wards were closer to hospitals than wealthier ones. This is probably best done, initially, by visual inspection of the geographical distribution hospitals and deprived areas. In this case, the large number of hospitals in what is a relatively small geographical area, taken with an inspection of the distribution of deprived wards and hospitals suggests that this is unlikely to have a major effect. Further analysis of 
this possibility would require detailed study of accessibility encompassing not only hospitals but also general practices and include examination of the means by which people come to hospitals and the ease of doing so. The work on the new resource allocation formula attempted to do this by including a variable on access to primary care, as well as variables relating to secondary NHS and private facilities. $^{2}$ More simple methods, such as crowfly distance or travel to work time are likely to be of limited relevance in this context.

It is also possible that the process of diagnosis is influenced by the social status of the patient, with doctors more likely to give different diagnoses for otherwise similar patients from wealthy and deprived backgrounds ${ }^{11}$ or to be otherwise influenced by what is most common in their clinical practice. ${ }^{12}$ A related effect could arise if a particular hospital had both a low admission threshold for a condition and a distinctive diagnostic practice, thus admitting a high proportion of people from a catchment area and labelling them in a particular way. The evidence on this point suggests that this is unlikely to explain the observed associations in this case study as the bias tends to be towards more specific diagnoses in the better off, who are investigated more thoroughly. ${ }^{13}$ It could, in theory, partly explain the lower level of association between deprivation and SERs for specific conditions compared with that seen for all causes, although this is not supported by the observation that, in this population, the Spearman's rank correlation between Carstairs index and SER for "other general conditions" was $-0.0521, \mathrm{p}=0.75$.

\section{HOW DOES DEPRIVATION AFFECT ADMISSION}

\section{RATES?}

If analysts can eliminate, as far as possible, these artifacts, there are three remaining possibilities. These are that those diseases categories showing a strong association with deprivation are either more common among the poor, that they are more likely to result in admission because of inadequate action in primary care in the detection or management of certain conditions, or that sick individuals from poor areas are more likely to be admitted because of their social circumstances.

The evidence for increased levels of ill health among the poor is strong, whether measured in terms of mortality, self limiting long standing illness, or a range of specific diseases, ${ }^{14}{ }^{15}$ with a growing body of evidence that material deprivation, rather than artefact, selection, or behavioural factors is the most important explanation. ${ }^{16}$ At a geographical level, for example, Carstairs and Morris $^{17}$ have demonstrated a socioeconomic gradient on the basis of postcode sectors in Scotland for death rates from diseases accounting for $70 \%$ of all deaths. The strongest gradients were found for bronchitis, lung and cervical cancer, pneumonia and cirrhosis. There is, in general, much less information on specific conditions that do not commonly result in death. Of course, a further complication arises from the growing body of evidence that the poor have less access to health care than would be justified by need ${ }^{18}$ and thus the observed hospitalisation rate is likely to underestimate the strength of the association between deprivation and morbidity. Consequently, this type of work should be linked, where possible, with studies of the prevalence of morbidity. Unfortunately, there are few such examples that are related directly to the need for services. ${ }^{1920}$

The second possibility, that high admission rates in deprived areas reflect failures of primary care, arises from the work of Billings et $a l^{11}$ in New York. He has identified conditions for which hospitalisation could be avoided by appropriate action at an early stage. These include asthma, diabetic ketoacidosis, congestive heart failure, and bacterial pneumonia. In this study, the association with deprivation for conditions such as asthma and bacterial pneumonia was less strong than for many other conditions and findings in the USA may not be applicable in the UK because of the presence of universal health care coverage here. A major study is under way in North Thames region to evaluate whether this is so ( $\mathrm{J}$ Dixon, personal communication) but evidence from a British study of stage at presentation of breast cancer ${ }^{2}$ found no difference between women from deprived and non-deprived backgrounds, unlike the situation in most American studies. Survival is, however, better in the women from less deprived backgrounds, suggesting that there are socioeconomic factors related to treatment or the response to it that are important.

The possibility that sick people living in poor areas are more likely to be admitted because of their social circumstances is intuitively attractive but there is little empirical work to support it.

It will never be possible at this level of analysis to provide all the answers. Rather, by highlighting the unexpected, it provides a pointer to the need for more detailed studies. The preceding explanations probably explain at least part of many of the observed differences. More importantly, along with several other methodological issues, they offer the basis for a research agenda. The remaining issues relate to the interpretation of findings and raise questions on both the classification of deprived areas and the categorisation of disease groups.

\section{CAVEATS}

Deprivation has many components such as lack of control and social and economic status. Census variables act as proxies for these different components, either individually or in combination. It is probable that for a specific condition an outcome such as the hospitalisation rate will be more closely associated with one of these underlying components than with a composite measure. For example, in the Whitehall study, employment grade (potentially a proxy for control) and car ownership (potentially a proxy for social status) contributed independently to all cause mortality. ${ }^{23}$ Furthermore, the use of a particular composite measure may give different results from another 
that reflects a different combination of underlying components. These components may act through both the likelihood of acquiring a condition and of obtaining treatment. In a comparison of four commonly used composite measures. ${ }^{24}$ there was little difference between the Carstairs and Townsend ${ }^{25}$ indices in the degree of association with a variety of admission rates but both were much more closely associated with admission rates than either the Jarman ${ }^{26}$ or Department of the Environment indices.

Potentially, any study based on wards is susceptible to the ecological fallacy, ${ }^{27}$ with results at a population level giving results different from those that would be obtained by studying individuals. Recent work at ward level by Sloggett and Joshi provides reassurance. They found that the excess mortality associated with residence in areas designated as deprived is wholly explained by the concentration therein of people with adverse socioeconomic factors.

The disease categories studied are clearly a function of the system used to aggregate conditions. Disease staging ${ }^{3}$ has advantages over some other approaches. It produces categories that have been shown to be well demarcated and clinically coherent, ${ }^{4}$ unlike, for example, ICD chapters or three digit codes. Unlike many alternative casemix systems, such as diagnosis related groups ${ }^{29}$ or healthcare resource groups ${ }^{30}$ it is not procedure driven. Systems that are would introduce a further element of clinical judgement and while they may be useful for further analysis of results from studies such as this, on their own they would only make interpretation more difficult. With disease staging ${ }^{3}$ it is possible in theory to conduct the analysis at a more detailed level by looking at differences in the stages within each category. These are ranked in terms of increasing severity and could be used to explore specific questions. They are not suitable for a blanket examination of the data as the numbers of cases in many stages are extremely small at this level of analysis.

The finding that none of the specific conditions is as closely associated with the Carstairs index as is total admissions is surprising. One possible explanation is that it reflects the addition of a random element of diagnostic variation for some conditions. This is an intrinsic limitation arising from the absence of explicit diagnostic criteria in the $\mathrm{ICD}^{31}$ and the known variation in the interpretation of clinical evidence. ${ }^{13}$ This interpretation is supported by the finding that the correlation for all other disease categories is still less than that for all cases.

In this case study, the association between Carstairs index and SER was not significant for bacterial pneumonia, cancer of the breast and colon, and head injury. The first and last of these are somewhat counter-intuitive and may reflect the use of a composite deprivation index that does not adequately capture aspects of deprivation directly relevant to the age groups involved most, the elderly and young respectively. Conversely, disease staging ${ }^{3}$ also highlighted two disease categories that are closely associated with deprivation levels that might otherwise be overlooked: functional digestive disorders and bursitis. The explanations for these associations are not immediately apparent. These findings indicate the scope for more detailed analysis by the commissioning agency.

\section{WHERE NOW?}

This case study using aggregate data inevitably raises more questions than it answers. It has many limitations, not least that it is simply a demonstration project commissioned by the Department of Health to explore how hospital episode data might be used at district level. As such, it offers a practical approach to what might be done, highlighting the pitfalls and challenges and, where possible, suggesting how they might be overcome. The extent to which these problems are important will depend on the characteristics of the district. The diversity seen throughout the country, in inner cities, suburbs, and rural areas with different travel distances, ethnic mixes, and economic differentials means that there is no such thing as a typical district. For the purposes of this case study, Kensington, Chelsea and Westminster district has the advantage of featuring a considerable amount, but not all this diversity within its boundaries. Consequently, the results cannot be generalised to other districts.

The study is also limited because the analyses undertaken are those that can reasonably be done by an information department at district level. Clearly much more could be learnt from much more sophisticated multi-level modelling although the skills and tools required are beyond the scope of most districts.

Further research is, however, required to inform those seeking to analyse geographical patterns of hospitalisation and deprivation, both in general and for specific conditions. The study examining the concept of ambulatory care sensitive conditions referred to above is an attempt at this. This information is needed urgently because of its important implications for the move to primary care led purchasing and the attempt to develop a capitation formula to fund general practitioners. Recent work by Sheldon et al concluded that it was not at present possible to produce such a formula. ${ }^{33}$ The present study adds a further dimension to this debate because of the widely differing hospital costs of the conditions studied. It is likely that general practitioners, albeit imperfectly, will be much better at predicting the probability of hospitalisation of a particular patient for a specific condition, on the basis of knowledge of the characteristics of the patient's clinical history and their socioeconomic status. Primary care led purchasing offers an open invitation for "cream-skimming" for those who wish to avail themselves of it and it will be very difficult for commissioning agencies, in their proposed regulatory role, to do anything about it. The example of health maintenance organisations in the USA illustrates what is likely to happen. ${ }^{34}$ Commissioning agencies, in the new regulatory role envisaged for them, face a 
formidable challenge if they are to monitor effectively the workings of the market.

This study was supported by the NHSME DISP Programme and was undertaken within the framework of the EU BIOMED
CAMISE project. Jennifer Dixon provided many helpful ideas CAMISE project. Jennifer Dixon provided many helpful ideas
and much practical information on the concept of ambulatory sensitive conditions. We are also grateful to Roy Carr-Hill and Colin Sanderson for helpful comments. The views expressed are those of the authors alone.

1 Department of Health. Working for patients. HMSO, London, 1989.

2 Smith P, Sheldon TA, Carr-Hill RA, Martin S, Peacock S, Hardman G. Allocating resources to health authorities: results and policy implications of small area analysis and use of inpatient services. BMF 1994;309:1050-4.

3 Gonnella JS, Hornbrook MC, Louis DZ. Staging of disease: A case-mix measurement. $\Im A M A$ 1984;251:637-44

4 McKee M, Petticrew M. Disease staging: A case mix system for purchasers: F Public Health Med 1993;15:25-36.

5 Carstairs V, Morris R. Deprivation and health in Scotland. Aberdeen, Aberdeen University Press, 1991.

6 SPSS. SPSS for Windows 6.0. Cary NY: SPSS Inc, 1989-93.

7 Microsoft Corporation. Microsoft Excel Version 5.0a. Redmond, WA: Microsoft, 1985-93.

8 Edwards N, Raftery J. Bedtime stories. Health Services $f$ 1995; 105:26-8. 9 Victor C. Under enumeration in the 1991 census. BMF

10 Clarke A, McKee M. The consultant episode: an unhelpful measure. BMf 1992;305:1307-8.

11 Samphier ML, Robertson C, Bloor MJ. A possible artefactual component in specific cause mortality gradients. $\mathcal{F}$ Epidemiol Community Health 1988;42:138-43.

12 Sutherland S. Irrationality. Harmondsworth: Penguin, 1994; $22-5$.

13 Davey Smith G, Leon D, Shipley MJ, Rose G. Socioeconomic differentials in cancer among men. Int $\mathcal{f}$ Epidemiol 1991;30:339-45.

14 DHSS. Inequalities in health: Report of a research working group. London: DHSS, 1980.

15 Blaxter M. Health and lifestyle. London: Tavistock, 1990.

16 Davey Smith G, Blane D, Bartley M. Explanations for socioeconomic differentials in mortality. Evidence from Britain economic differentials in mortality. Evidence from B

17 Carstairs V, Morris R. Deprivation and health in Scotland. Health Bull Edin 1990;48:162-75.
18 Tudor Hart J. The inverse care law Lancet 1971;i:405-12. 19 Harries U, Landes R, Popay J. Visual disability among older people: a case study in assessing needs and examining services. F Public Health Med 1994;16:211-18.

20 Hunter DJW, McKee CM, Black NA, Sanderson CFB. Urinary symptoms: prevalence and severity in British men aged 55 and over. $\mathcal{f}$ Epidemiol Community Health 1994;48 569-75.

21 Billings J, Zeitel L, Lukomnik J, Carey TS, Blank AE, Newman L. Impact of socioeconomic status on hospital use in New York City. Health Affairs 1993;12:162-73.

22 Carnon AG, Ssemwogerere A, Lamont DW, Hole DJ, Mallon AA, George WD, Gillis CR. Relation between socioeconomic deprivation and pathological prognostic factors economic deprivation and pathological prognostic factor

23 Davey Smith G, Shipley MJ, Rose G. The magnitude and causes of socio-economic differentials in mortality: further evidence from the Whitehall study. $\mathcal{F}$ Epidemiol Community Health 1990;44:265-70.

24 Campbell DA, Radford JMC, Burton P. Unemployment rates: an alternative to the Jarman index? $B M \mathcal{F} 1991 ; 303$ 750-5.

25 Townsend P, Simpson D, Tibbs N. Inequalities in health in the city of Bristol: a preliminary review of statistical evidence. Int f Health Services 1985;15:637-63.

26 Jarman B. Underprivileged areas: validation and distribution of scores. BMf 1984;289:1587-92.

27 Robinson WS. Ecological correlations and the behavior of individuals. American Sociological Reviews 1950;15:351-7.

28 Sloggett A, Joshi H. Higher mortality in deprived areas: Sloggett $A$, Joshi $H$. Higher mortality in deprived areas:
community or personal disadvantage? $B M \mathcal{F} 1994 ; 309$ : community $1470-4$.

29 Fetter RB, Shin Y, Freeman JL, Averill RF, Thompson JD. Case mix definition by diagnosis related groups. Medical Care $1980 ; 18$ (2 suppl): $1-53$.

30 Buckland RW. Healthcare resource groups: A more sensitive and less costly approach to contracting. BMf 1994;308: 1056.

31 Iezzoni LI. Using administrative diagnostic data to assess the quality of hospital care: Pitfalls and potential of ICD9CM. International fournal of Technology Assessment in Health Care 1990;6:272-81.

32 Koran LM. The relationship of clinical methods, data and judgements. N Engl Med $\mathcal{F}$ 1975;293:695-701.

33 Sheldon TA, Smith P, Borrowitz M, Martin S, Carr-Hill RA. Attempt at deriving a formula for setting general RA. Attempt at deriving a formula for setting general
practitioner fundholding budgets. BMF 1994;309:105964 .

34 Light DW. Managed care: false and real solutions. Lancet 1994;344:1197-9. 